Proc. XIX International School of Semiconducting Compounds, Jaszowiec 1990

\title{
INTRA-SHALLOW-DONOR PHOTOCONDUCTIVITY IN SEMI-INSULATING
} $\mathrm{GaAs}^{*}$

\author{
K. KARPIERZ, \\ Institute of Experimental Physics, Warsaw University, Hoża 69,00-681 Warsaw, Poland
}

AND M.L. SADOWSKI

High Pressure Research Centre, Polish Academy of Sciences, Warsaw, Poland (Received August 8, 1990)

The far-infrared photoconductivity of a semi-insulating GaAs sample was measured at $4.2 \mathrm{~K}$ as a function of magnetic field up to $7 \mathrm{~T}$. Apart from a peak corresponding to the $1 s-2 p_{+1}$ transition of the hydrogen-like shallow donor, a well-pronounced structure was observed which does not appear in n-type GaAs.

PACS numbers: 72.40.+w, 78.20.ls, 78.50.Ec,Ge

The far-infrared (FIR) magnetophotoconductivity of $n$-type GaAs has been extensively studied by many authors (see e.g. [1]). To our knowledge, however, there have been no reports of such investigations for semi-insulating (SI) GaAs. The physical situation of a hydrogen-like donor in this material differs from the $n$-type on at least two counts: first, the donor states are empty at low temperatures due to the large compensation, and second, because of the lack of screening, the ionized impurity potentials give rise to extremely strong local electric fields.

The experimental set-up consisted of a FIR molecular laser and a $7 \mathrm{~T}$ superconducting magnet (for details see [2]). For this experiment a monochromatic source of near-band-gap light was added. The sample [3] was cooled down in the dark and then illuminated with infrared (IR) light $(\lambda \sim 0.86 \mu \mathrm{m})$ from the monochromator. This wavelength was chosen to avoid metastable phenomena connected with intra-EL2 absorption, which takes place in the range $0.95 \mu \mathrm{m}<$ $\lambda<1.2 \mu \mathrm{m}$ [4]. In this way a stable population of neutral shallow donors was obtained (see Fig. 1a). This was possible because electrons pumped from deeper

"This work was supported in part by CPBP 01.05 
(a)
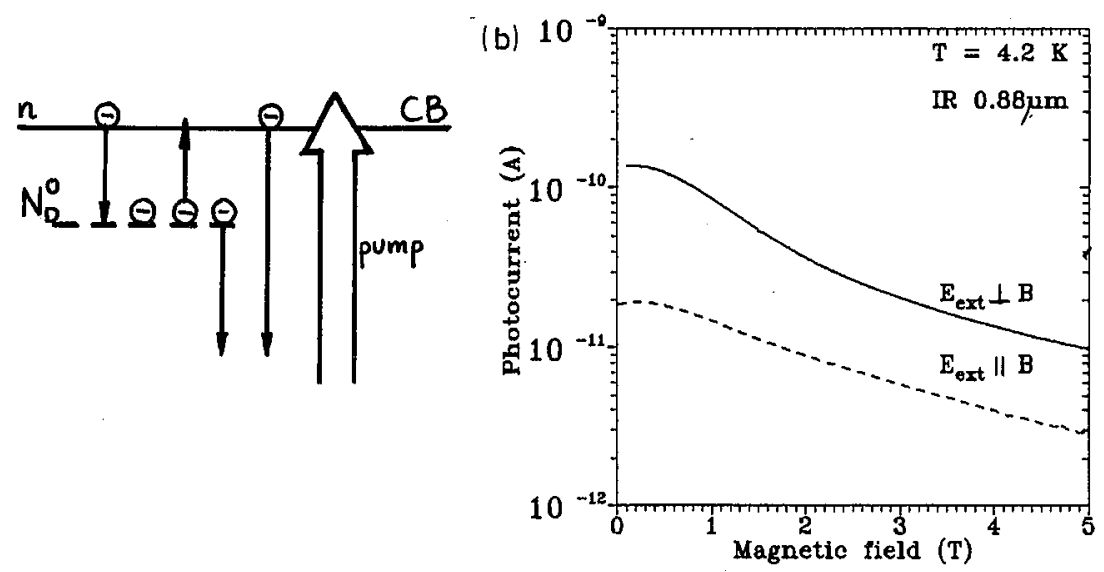

Fig. 1. a) A schematic representation of the processes occurring in an SI GaAs sample illuminated with near band-gap light. Electrons are pumped into the conduction band from states in the band gap, i.e. from the EL2 defect and acceptors (large arrow). Some drop back immediately, others populate empty donor states. Electrons from donor states can be thermally excited into the conduction band, or drop down to lower levels. After a certain time, a dynamic equilibrium between all these processes is achieved. b) Current through the sample as a function of the magnetic field, for two configurations of the sample: $\boldsymbol{E}_{\text {ext }} \perp \boldsymbol{B}$ (solid line), and $\boldsymbol{E}_{\text {ext }} \| \boldsymbol{B}$ (dashed line). The voltage across the sample was constant $U=0.3 \mathrm{~V}$. IR signifies the wavelength of the incident infrared light.

levels in the band gap to the shallow donor via the conduction band are probably displaced in configuration space and cannot easily return to their former states. Additionally, some free electrons always exist in the conduction band; as a result, the conductivity of the sample rises by a few orders of magnitude.

With the electron distribution in its dynamic steady state, the DC conductivity $\sigma$.of the sample was measured as a function of the magnetic field for two different configurations (Fig. 1b). In the first, the electric field across the sample $\left(\boldsymbol{E}_{e x t}\right)$ was perpendicular to the magnetic field $(\boldsymbol{B})$; in the second, the two fields were parallel. The character of the $\sigma(B)$ curve is the same for both cases, and the magnitude of the change is only slightly larger when $E_{\text {ext }} \perp B$. The magnetic field dependent conductivity may be written in the form

$$
\sigma(B)=n(B) e \mu(B)
$$

where $n(B)$ is the free carrier concentration, $e$ is the electron charge and $\mu(B)$ describes the effective mobility of an electron in the conduction band.

When the sample is additionally illuminated with FIR laser light, the resulting changes in the conductivity can be written as

$$
\Delta \sigma(B)=\Delta n(B) e \mu(B) .
$$



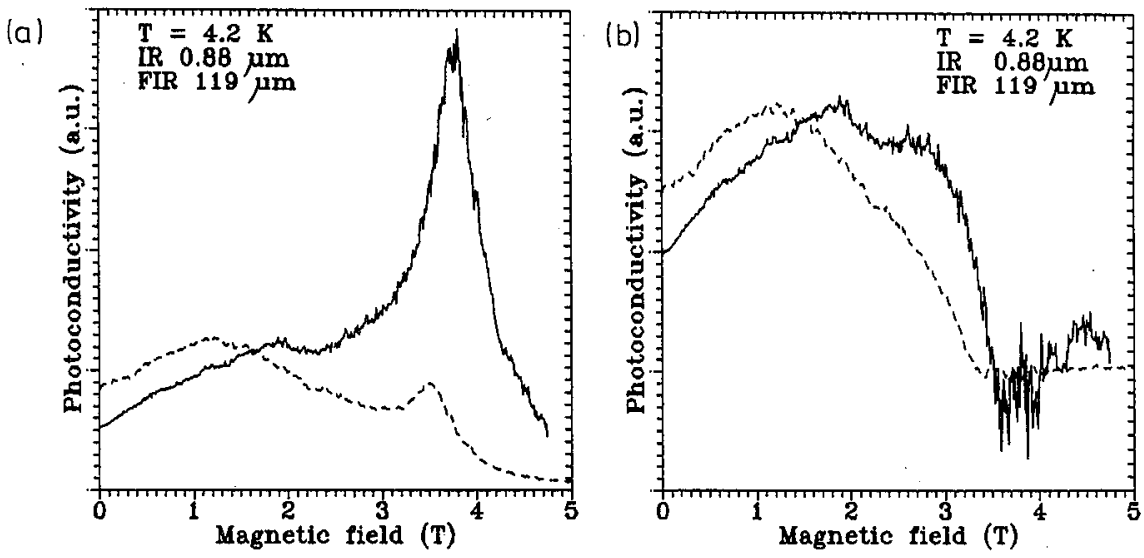

Fig. 2. a) Photoconductivity spectra $\Delta \sigma / \sigma$ as a function of the magnetic field, for two configurations of the sample: $\boldsymbol{E}_{\text {ext }} \perp \boldsymbol{B}$ (solid line), and $\boldsymbol{E}_{\text {ext }} \| \boldsymbol{B}$ (dashed line). b) The same spectra, after numerical subtraction of the $1 s-2 p_{+1}$ peaks. The curves were not smoothed numerically. The peaks were assumed to be symmetric and were fitted with a Lorentz curve. The voltage across the sample was constant. IR and FIR signify the wavelengths of the incident infrared and far infrared light, respectively.

We can see that dividing $\Delta \sigma(B)$ by $\sigma(B)$ rids us of the unknown quantity $\mu(B)$, yielding

$$
\Delta \sigma(B) / \sigma(B)=\Delta n(B) / n(B) .
$$

Figure 2a shows such tracings of two runs in two different configurations. It can be seen that in both cases there is a clearly visible peak in the vicinity of $3.6 \mathrm{~T}$, which corresponds well to the $1 s-2 p_{+1}$ transition in $n$-type GaAs [1]. The half-width of this peak is about $0.5 \mathrm{~T}$, which is about twice more than usual for a pure $n$-type. Moreover, there is a wide structure at low magnetic fields, which does not appear in the latter material. This structure qualitatively resembles one observed in FIR absorption measurements [5], which was attributed by the authors to shallow donors with an increased binding energy due to the strong local electric fields in the sample. When the $1 s-2 p_{+1}$ peak is subtracted for both configurations it can be seen that the low magnetic field structures appear to be centered at different values of $B$ (Fig.2b). Assuming that photoconductivity takes place in the conduction band only, the influence of absorption by acceptors and holes can be neglected in the interpretation of our results. Furthermore, due to mixing of states caused by local electric fields, the breaking of selection rules is highly probable. The difference in the positions of the structure for the two configurations is difficult to explain only by a change of the selection rules. We therefore suggest an anisotropy of the state responsible for the transitions appearing at low magnetic field. The nature of this state (or states) is unknown. One possible explanation is the formation of direction-sensitive donor pairs. A specific direction 
could be singled out by either the crystallographic structure or the direction of crystal growth.

Moreover, the similar behaviour of transverse and longitudinal magnetophotoconductivity (Fig.1b) suggests a more complicated conduction mechanism than simple conduction band transport phenomena.

The authors wish to express their gratitude to Prof. M. Grynberg for many helpful discussions.

\section{References}

[1] G.E. Stillman, C.M. Wolfe, J.O. Dimmock, in: Semiconductors and Semimetals, Vol. 12, ed. R.K. Willardson, A.C. Beer, Academic Press, 1977.

[2] K. Pastor, R. Triboulet, Phys. Status Solidi A 100, K51 (1987).

[3] The sample was a rectangular slab $(0.5 \times 1.0 \times 3.0 \mathrm{~mm})$ of bulk SI GaAs (grown by the LEC technique in the (100) direction) with a concentration of the EL2 defect about $10^{16} \mathrm{~cm}^{-3}$. The dark resistance at $4.2 \mathrm{~K}$ was of the order of $10^{12} \Omega$. Two nonrectifying Au-Ge-Ni contacts were alloyed by Dr Kamińska, and Dr Piotrowska from the Institute of Electron Technology in Warsaw.

[4] F. Fuchs, B. Dishler, Appl. Phys. Lett. 51 (25), 2115 (1987).

[5] D. Paget, P.B. Klein, Phys. Rev. B 34, 971 (1986). 